\title{
miR-503 Inhibits Proliferation, Migration, And Angiogenesis Of Glioma By Acting On VEGFA Through Targeting LRIG2
}

This article was published in the following Dove Press journal: Cancer Management and Research

\author{
Sheng-Li Sun \\ Yu-Gao Shu \\ Mei-Yi Tao
}

Department of Neurosurgery, Hunan Provincial People's Hospital, Changsha 410005, People's Republic of China
Correspondence: Mei-Yi Tao Department of Neurosurgery, Hunan Provincial People's Hospital, No. 6I, Jiefang West Road, Changsha 410005, Hunan Province, People's Republic of China

Tel +86 I37 2389378 I

Email taomeiyirqc@163.com
Background: Glioma is a common malignant tumor of the human central nervous system, and the pathological characteristics include invasive growth, angiogenesis, and so on. Ectopic expression of miR-503 works as a critical factor in cancer cell proliferation, migration, and capillary-like tube formation. The potential mechanisms of miR-503 in angiogenesis of glioma cells are still not reported.

Methods: The expression levels of miR-503, LRIG2, and VEGFA mRNA and protein were performed by quantitative reverse transcription-PCR or Western blot assay. Dual-Luciferase reporter gene assay was used to determine the interaction between miR-503 and LRIG2. The concentration of VEGFA was measured using the ELISA method. The cell proliferation, migration, and angiogenesis of cocultured HCMEC/D3 cells were analyzed by MTT assay, transwell detection, and tube formation assay, respectively.

Results: The expression levels of LRIG2 and VEGFA were reduced in glioma cells with miR-503 overexpression and enhanced with miR-503 inhibition. Moreover, cell proliferation, migration, and angiogenesis of cocultured HCMEC/D3 cells were alleviated with miR-503 mimics transfection. VEGFA and miR-503 inhibitor promoted cell proliferation, cell migration, and angiogenesis. Luciferase reporter gene assay revealed that miR-503 could directly target LRIG2. Furthermore, knockdown of LRIG2 or addition of VEGF inhibitor bevacizumab could abrogate the effect of miR-503 inhibitor on VEGFA expression, as well as the promotion of cell proliferation, migration, and angiogenesis.

Conclusion: MiR-503 mediated LRIG2 suppression and regulated the expression of VEGFA, thereby reducing cell proliferation, migration, and angiogenesis of glioma cells. These results provide new insight into the action mechanism of miR-503-modulated signaling pathway in angiogenesis of glioma cells.

Keywords: glioma, miR-503, LRIG2, VEGFA, angiogenesis

\section{Introduction}

Glioma is regarded as the most common malignant tumor of the human central nervous system, and glioblastoma accounts for about $80 \%$ of malignant brain tumors, which is characterized by aggressive angiogenesis. ${ }^{1}$ Glioma has high invasion, recurrence, and mortality rate because the glioma cells growth and proliferation has considerably dependence on angiogenesis. Angiogenesis is regulated by the interaction of proangiogenic and antiangiogenic factors. The antiangiogenic factors can be generated by glioma cells and act on glioma-associated endothelial cells, thus regulating the process of angiogenesis. ${ }^{2}$ It has been demonstrated that abnormal lncRNAs and miRNAs 
expression, as well as interaction between each other has remarkable effect in glioma angiogenesis. ${ }^{3}$ Therefore, the elucidation of critical factors and signaling pathways involved in antiangiogenesis becomes an important therapy in glioma treatment.

Leucine-rich repeats and immunoglobulin-like domains protein 2 (LRIG2) gene encodes a transmembrane protein, which contains leucine-rich repeats and immunoglobulinlike domains. LRIG2 is produced in the human cytoplasm of glioma cells and localizes to the cell surface, perinuclear and cytoplasmic compartments. ${ }^{4}$ The previous study has demonstrated that the downregulated expression of LRIG2 inhibits proliferation, increases cell spontaneous apoptosis in glioblastoma cell GL15, which provides a new target for glioma treatment. ${ }^{5}$ Moreover, the reduced expression of LRIG2 can inhibit the angiogenesis of glioma cells by decreasing the expression of epidermal growth factor receptor (EGFR) as well as vascular endothelial growth factor A (VEGFA). ${ }^{6}$

miRNAs are endogenous noncoding RNAs, consisting of 18 to 24 nucleotides, which plays inhibition or promotion effects on cell proliferation, apoptosis, differentiation, induction of angiogenesis, and activation of metastasis and invasion. ${ }^{7}$ The recent studies have been identified that the abnormal expression of miRNAs is closely related to cell proliferation, apoptosis, and drug resistance in glioblastoma. ${ }^{8}$ It has been proved that miR-503 acts as a repressor in glioma pathogenesis by targeting L1 cell adhesion molecule (L1CAM). ${ }^{9}$ The mechanism of miR-503 on the angiogenesis of glioma and the associated signaling pathway has still not been reported. But previous studies have revealed that miR503 plays a tumor inhibition role in several cancers, such as hepatocellular carcinoma (HCC), which suppressed the growth and angiogenesis of HCC cells by directly targeting fibroblast growth factor-b (FGF2) and VEGFA. ${ }^{10,11}$ FGF2 and VEGFA have been shown to be involved in the regulation of angiogenesis, which are angiogenic factors suppressing deregulated blood vessel formation in multiple diseases such as cancer. ${ }^{12,13}$ According to the bioinformatics prediction, we found that miR-503 has the putative binding sites targeting LRIG2. It is speculated here that miR-503 may play an important role in glioma angiogenesis by targeting LRIG2.

In this study, the possible molecular mechanism underlying the regulatory effect of miR-503 on VEGFA through targeting LRIG2 during glioma angiogenesis is presented. In general, our research provides a new evidence that the miR-503-modulated suppressing of LRIG2 is responsible for the transcription downregulation of VEGFA, thus inhibiting the angiogenesis of glioma cells.

\section{Materials And Methods Cell Culture}

The human glioma cell lines (A172 and U251) and immortalized human cerebral microvascular endothelial cell line D3 (HCMEC/D3) were obtained from Chinese Academy of Sciences (Shanghai, China). All of the above cell lines were selected and cultured in DMEM (purchased from Gibco, USA), with 10\% FBS (Invitrogen, CA, USA) supplementation. The cells were maintained in an incubator at $37^{\circ} \mathrm{C}$ with humidified $5 \% \mathrm{CO}_{2}$.

\section{Cell Transfection}

The shRNAs targeting LRIG2 (sh-LRIG2) were designed and synthesized by GenePharma (Shanghai, China). The miR-503 mimics, miR-503 inhibitor, respective negative control miRNA (miR-NC), and inhibitor NC were purchased from RiboBio (Guangzhou, China). Then, they were transfected into A172 and U251 cells using Lipofectamine 2000 reagent (Invitrogen) according to the manufacturer's instructions. After the transfection, the cells were cultured and applied for further experiment assay.

\section{Luciferase Reporter Assay}

The wild-type (WT) fragments from LRIG2 (LRIG2-WT) containing the potential binding sites and corresponding mutant type (MUT) fragments (LRIG2-MUT) of miR-503 were cloned into the pMIR-report plasmid vector (Promega, USA) to generate the LRIG2 luciferase reporter construct. Then, the vectors and miR-503 mimics were cotransfected into A172 and U251 cells using Lipofectamine 2000 reagent (Invitrogen). Subsequently, the cells were harvested, lysed and the relative Rluc/Luc ratio was measured as described previously by the Dual-Luciferase reporter gene system (Promega) in accordance with the protocol. $^{14}$

\section{Total RNA Extraction And Quantitative Reverse Transcription-PCR (qRT-PCR)}

Total RNA was extracted using TRIZOL reagent (Sigma, USA) following the manufacturer's protocol from the indicated cultured cell lines. The relative expression levels of miR-503, VEGFA, and LRIG2 were detected by qRTPCR assay using the SYBR-Green qPCR SuperMix 
(Invitrogen) according to the instructions. The glyceraldehyde-3-phosphate dehydrogenase (GAPDH) was served as the endogenous control. Gene-specific primers of miR-503, VEGFA, and LRIG2 for qRT-PCR analyses were listed as follows: miR-503-F, 5'-CCTATTTCCCATGATTCCTTC ATA-3' and miR-503-R, 5'-GTAATACGGTTATCCACGCG -3'; VEGFA-F, 5'-CAAGGCCAGCACATAGGAGA-3' and VEGFA-R， 5'-ACGCGAGTCTGTGTTTTTGC-3'; LRIG2F, 5'-TTGCTGAGGAGGACGTTCTTG-3' and LRIG2-R, 5'TGTGGCAGGACATGGAAGAAG-3'; GAPDH-F, 5'-CTG ACATGCCGCCTGGAGA-3' and GAPDH-R, 5'-ATGTAGG CCATGAGGTCCAC-3'. U6-F, CGCTTCGGCAGCACATA TACTAAAATTGGAAC, and U6-R, GCTTCACGAATTTG CGTGTCATCCTTGC. The PCR procedure used in this study was as follows: $95^{\circ} \mathrm{C}$ for $5 \mathrm{mins}$, followed by $94^{\circ} \mathrm{C}$ for $40 \mathrm{~s}$, $62^{\circ} \mathrm{C}$ for $60 \mathrm{~s}$ and $72^{\circ} \mathrm{C}$ for $60 \mathrm{~s}$, with a total of 40 cycles, and at last $72^{\circ} \mathrm{C}$ for 10 mins.

\section{Protein Extraction And Western Blot}

The cultured cells were lysed using the RIPA reagent (Millipore, USA) and the concentration of the proteins was quantified by the BCA protein assay kit (Bio-Rad, USA). The samples were subjected to $8 \%$ SDS-PAGE for electrophoresis and transferred onto the polyvinylidene fluoride membranes (Millipore). Then, the membrane was blocked with 5\% skimmed milk diluted in Tris-buffered saline (TBS) containing $0.1 \%$ of Tween 20 (TBST buffer) for $1 \mathrm{hr}$ at room temperature. The protein-specific primary antibodies of anti-VEGFA, anti-LRIG2, and anti-GAPDH (Abcam, USA) were added, then the protein was incubated at $4^{\circ} \mathrm{C}$ overnight. Subsequently, the membranes were rinsed by TBST buffer and probed with secondary horseradish peroxidase (HRP) conjugated antibodies for $1 \mathrm{hr}$ at room temperature. Then, the proteins were determined by the enhanced chemiluminescence reagent (Millipore) in accordance with the manufacturer's protocol and the bands were quantified using the ImageJ software.

\section{ELISA}

To investigate the concentration of VEGFA in the tumorconditioned medium (TCM), HCMEC/D3 cells were cocultured with the human glioma cell lines A172 and U251 to establish the coculture system. ${ }^{15,16}$ The respective TCM from either transfected mono-glioma cell or coculture system of HCMEC/D3 cells was prepared, and the supernatant of the cells was harvested and centrifuged at $4^{\circ} \mathrm{C}$ for 20 mins at $2000 \times \mathrm{g}$ to remove the cell debris, then it was stored at $-80^{\circ} \mathrm{C}$ for further use. After dilution, the concentration of VEGFA in cell supernatants was quantitatively detected using the Human VEGFA ELISA kit (R\&D Systems, USA) following the manufacturer's instruction. The OD value was read at $450 \mathrm{~nm}$ using the Flexstation 3 microplate reader. The purified VEGFA was used for creating a standard curve, and the results were analyzed by CurveExpert 1.3 software program.

\section{MTT Assay}

To measure the cell proliferation ability of HCMEC/D3 cells induced by coculture with glioma cells, MTT assay was carried out using the coculture system. An MTT regent of $40 \mathrm{mg} / \mathrm{mL}$ was added to each culture medium and incubated for $4 \mathrm{hrs}$. Then, the supernatants were removed and the reaction was terminated. The formazan product was dissolved in dimethyl sulfoxide, and the absorbance value was measured using the microplate reader at $570 \mathrm{~nm}$.

\section{Transwell Assay}

Following transfection, the cell migration ability of HCMEC/D3 cells induced by coculture with glioma cells was determined by transwell assay using the coculture system ( $8 \mu \mathrm{m}$ pore size) as described before. On the lower transwell chamber, $1 \times 10^{5} /$ well of A172 or U251 cells were seeded for $24 \mathrm{hrs}$, then cocultivation with $1 \times 10^{5} /$ well of HCMEC/D3 cells were seeded in the upper transwell chamber. After $24 \mathrm{hrs}$ incubation, HCMEC/D3 cells on the upper surface of the fibronectincoated polycarbonate membrane were removed. The migrated cells were fixed with $10 \%$ formalin at room temperature and stained with $0.1 \%$ crystal violet. Then, the migrated cells were counted from five different fields of each filter under a microscope.

\section{Tube Formation Assay}

To explore the tube formation ability of HCMEC/D3 cells induced by coculture with glioma cell, cothe culture system $(0.4 \mu \mathrm{m}$ pore size) was used for the tube formation assay. Fifty microliters of diluted matrigel (BD Biosciences, USA) was prepared and added to HCMEC/ D3 cells in the upper transwell chamber. Subsequently, the cells were incubated at $37^{\circ} \mathrm{C}$ overnight. On the lower transwell chamber, $1 \times 10^{5} /$ well of A172 or U251 cells were seeded, then cocultivated with HCMEC/D3 $\left(1 \times 10^{5}\right.$ / well) in the upper chamber. After $24 \mathrm{hrs}$ incubation, the branch points of formed capillary-like structures, which represent the angiogenesis degree in vitro, were observed 
and photographed from five different fields of each filter using an inverted microscope to assess the tube formation ability of HCMEC/D3 cells.

\section{Statistical Method}

All the data of this study are statistical analyzed by SPSS 21.0 statistical software. The measurement data were expressed as mean $\pm \mathrm{SD}$. The Student's $t$-test was used for pairwise comparison and single-factor ANOVA was used for multigroup comparison. $\mathrm{P}<0.05$ represents the difference was statistically significant.

\section{Results}

\section{miR-503 Inhibits Angiogenesis In Glioma Cells}

In order to investigate the molecular regulation mechanism of miR-503 in the angiogenesis of glioma cells, miR-NC, miR-503 mimics, inhibitor NC, and miR-503 inhibitor transfection were carried out in human glioma cell lines A172 and U251 for further analysis. To compare the expression levels of miR-503 and VEGFA mRNA in glioma cells, qRT-PCR assay was performed in A172 and U251 cells. As shown in Figure 1A, the miR-503 mRNA expression was increased in the miR-503 mimics group and decreased in the miR-503 inhibitor group compared with that in the miRNC group or inhibitor NC group. The VEGFA levels presented the opposite results (Figure 1A). In addition, Western blot assay results were in accordance with qRT-PCR, which showed that the expression of VEGFA protein was inhibited in cells with miR-503 mimics transfection and promoted in miR-503 inhibitor group (Figure 1B). Moreover, the concentration of VEGFA was quantified by ELISA. Similarly, it showed that the overexpression of miR-503 reduced VEGFA secretion and miR-503 inhibitor increased VEGFA concentration (Figure 1C). To further determine the cell proliferation ability of HCMEC/D3 cells induced by coculture with glioma cells, MTT assay was performed using the coculture system. It was revealed that the cell proliferation ability was obviously elevated in cells with miR-503 inhibitor transfection than that in cells with miR503 mimics transfection (Figure 1D). Moreover, through the transwell assay, it was found that the overexpression of miR-503 gene markedly reduced the migration capacity of HCMEC/D3 cells compared with cells in miR-NC group (Figure 1E). The miR-503 inhibitor significantly enhanced the migration capacity of HCMEC/D3 cells compared with cells in the inhibitor NC group (Figure 1E). Besides, the angiogenesis capacity of HCMEC/D3 cells was higher with miR-503 inhibitor transfection compared with the inhibitor $\mathrm{NC}$ group, and that in cells with miR-503 mimics transfection was weaker than the miR-NC group (Figure 1F). These results indicated that miR-503 played a negative regulation role in the angiogenesis of glioma cells.

\section{miR-503 Directly Targets LRIG2 And Negatively Regulates Its Expression}

As shown in Figure 2A, it was identified that the region of miR-503 has putative binding sites for LRIG2 by the bioinformatics analysis. Luciferase reporter assay revealed that the luciferase activities were inhibited significantly when LRIG2-WT was co-transfected with miR-503 mimics, while no significant change was found in cells with LRIG2MUT (Figure 2B). These results suggested that there was binding activity of miR-503 to LRIG2 in glioma cells. As shown in Figure $2 \mathrm{C}$, transfection with miR-503 mimics decreased LRIG2 mRNA expression in A172 and U251 cells and miR-503 inhibitor increased the expression levels of LRIG2 (Figure 2C). Moreover, the results of Western blot assay were similar to that of the qRT-PCR (Figure 2D). Taken together, in the glioma cells, miR-503 regulated LRIG2 expression by directly binding to the related sites.

\section{Downregulation Of miR-503 In Glioma Cells Induces Tumor Angiogenesis Via Targeting LRIG2}

To further explore the cross-talk of miR-503 and LRIG2 in tumor angiogenesis, the expression levels of LRIG2 and VEGFA mRNA and protein were determined following transfection with inhibitor NC, miR-503 inhibitor and cotransfection of sh-LRIG2 and miR-503 inhibitor. As expected, miR-503 inhibitor significantly induced LRIG2 and VEGFA mRNA and protein expression, whereas such effects were lost by co-transfection of sh-LRIG2 and miR-503 inhibitor. (Figure 3A and B). Similarly, the level of VEGFA in A172 and U251 cells with miR-503 inhibitor transfection was elevated, and this effect was attenuated when the cells were cotransfectionof sh-LRIG2 and miR-503 inhibitor (Figure 3C). To investigate the possible regulation mechanism of miR-503regulated LRIG2 expression in cell proliferation, migration, and tumor angiogenesis, the transwell coculture model was used. The MTT assay demonstrated that the induction effect of HCMEC/D3 cell viability in cells transfected with miR-503 inhibitor was reversed after co-transfection of sh-LRIG2 and miR-503 inhibitor (Figure 3D). Furthermore, the inhibition of 

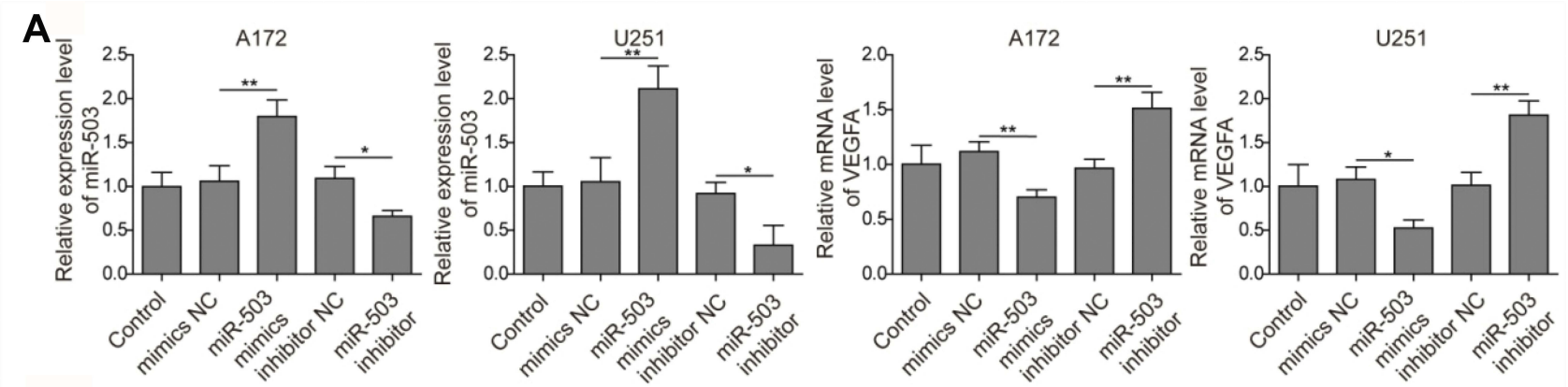

B

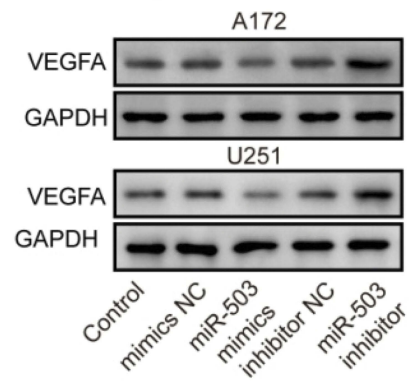

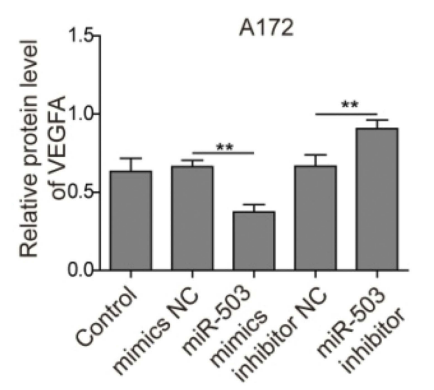

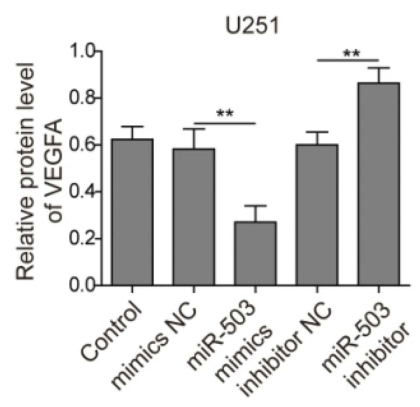

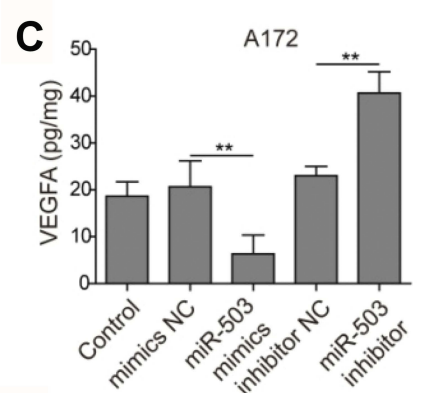
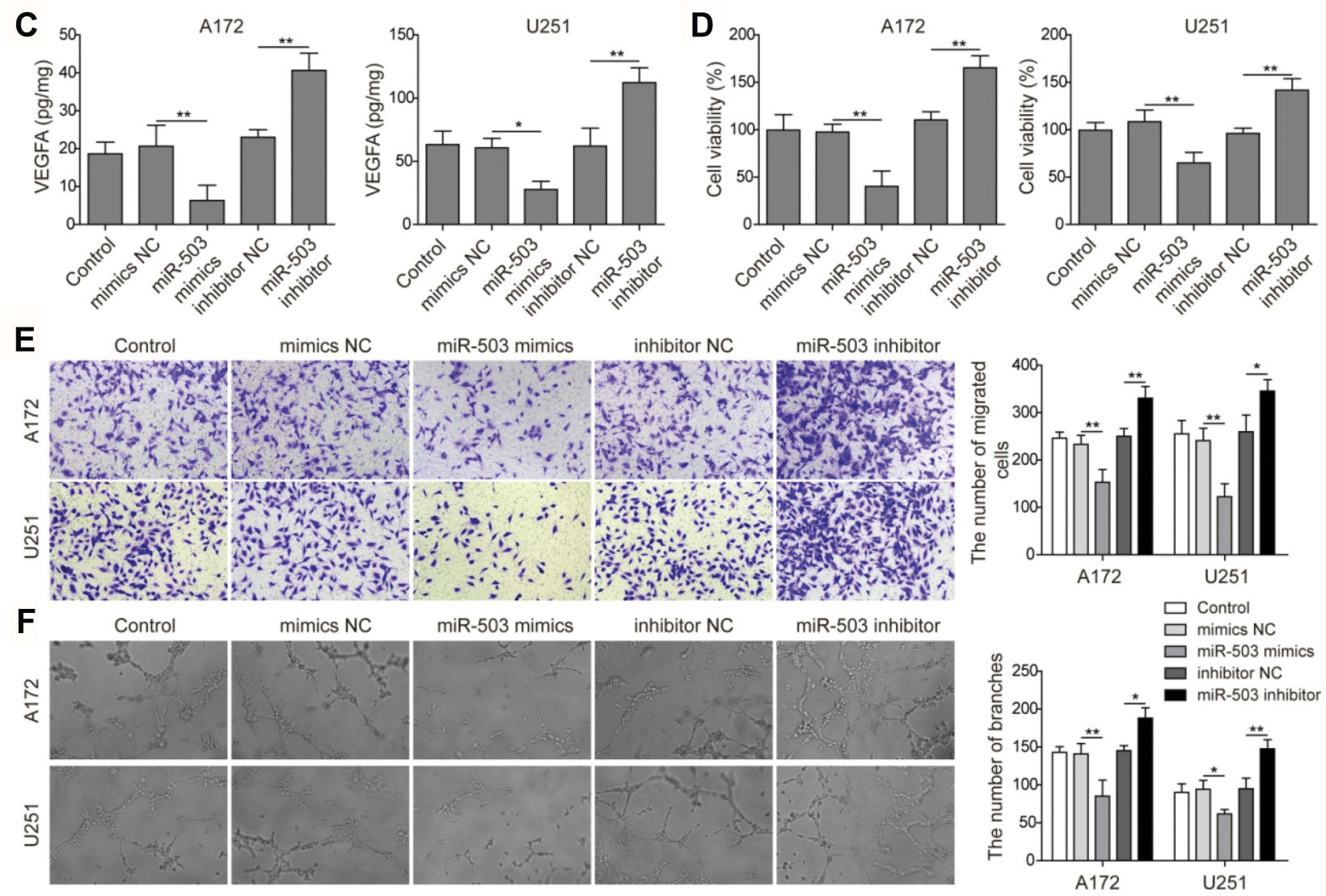

Figure I The effects of miR-503 on expression levels of VEGFA in AI72 and U25 I cells, and cell proliferation, cell migration and angiogenesis of coculture HCMEC/D3 cells. (A) The miR-503 and VEGFA transcription levels were detected by qRT-PCR in AI72 and U25I cells. (B) Western blot analysis of VEGFA expression in AI72 and U25I cells. (C) ELISA assay was performed to detect VEGFA concentration using the cocultured system. (D) HCMEC/D3 proliferation induced by co-culturing with glioma cells was assessed by the MTT method using the cocultured system. (E) The migration ability of cocultured HCMEC/D3 cells was detected by transwell assay. (F) HCMEC/D3 angiogenesis ability in cells after indicated treatment. The experiment was repeated for three times. Data are the mean \pm SD for three independent experiments. P value was determined by one-way ANOVA following Tukey's post hoc test. $* \mathrm{P}<0.05 ; * * \mathrm{P}<0.01$.

miR-503 was able to induce migration activities of HCMEC/ D3 cells, but this effect could be partially abrogated by cotransfection with sh-LRIG2 (Figure 3E). As shown in
Figure $3 \mathrm{~F}$, tube formation ability was obviously elevated in HCMEC/D3 cells with miR-503 inhibitor and the cotransfection of sh-LRIG2 and miR-503 inhibitor inhibited 

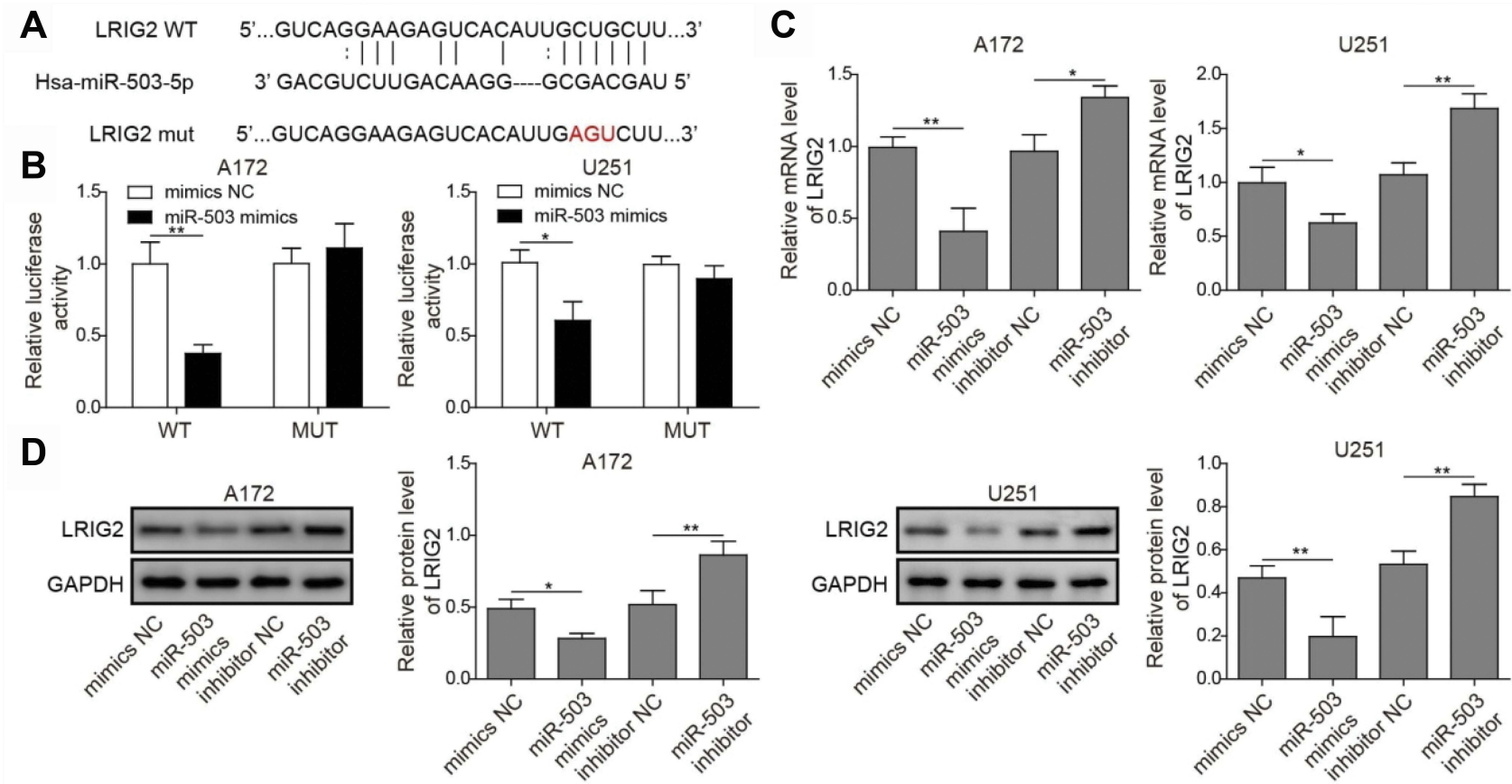

Figure 2 Detection of interaction between miR-503 and LRIG2. (A) miR-503 and its predicted sites in LRIG2 sequence. (B) The luciferase activity was detected for determining the binding of miR-503 to the predicted sites of LRIG2. (C) qRT-PCR detection of LRIG2 mRNA expression in glioma cells transfected with miR-503 mimics and miR-503 inhibitor. (D) The expression of LRIG2 protein was analyzed by Western blot after indicated treatment. The experiment was repeated for three times. P value was determined by ANOVA following Tukey's post hoc test. Data are the means \pm SD for three independent experiments. *P $<0.05 ; * * P<0.0$ I.

the induction of angiogenesis (Figure 3F). Based on these results, the tumor angiogenesis effects induced by miR-503 inhibition are partially mediated by targeting LRIG2.

\section{miR-503 Regulates Tumor Angiogenesis Via LRIG2-Regulated VEGFA Expression}

To investigate the underlying mechanisms of miR-503/ LRIG2/VEGFA in tumor angiogenesis, HCMEC/D3 cells in the presence of $20 \mathrm{ng} / \mathrm{mL}$ of recombinant VEGFA protein, transfected with miR-503 inhibitor, co-transfected with miR503 inhibitor and sh-LRIG2, or VEGF inhibitor bevacizumab were evaluated. The concentration of in the culture medium after coculture was detected, and it was revealed that in the presence of recombinant VEGFA protein and miR503 inhibitor, VEGFA accumulation was elevated, and in miR-503 inhibitor + sh-LRIG2 or miR-503 inhibitor + bevacizumab group, the effects were abrogated (Figure 4A). The transwell coculture model was used to assess the cell proliferation, cell migration, and angiogenesis. It was found that, VEGFA and miR-503 inhibitor promoted HCMEC/D3 cell proliferation, cell migration, and angiogenesis. Nevertheless, miR-503 inhibitor + sh-LRIG2 and miR-503 inhibitor + bevacizumab groups alleviated the effects (Figure 4B-D). These results suggested that miR-503 was involved in regulating the tumor angiogenesis, possibly through the VEGFA expression regulated by LRIG2.

\section{Discussion}

Illuminating the molecular regulation mechanisms underlying antiangiogenesis in glioma is regarded as an important approach to discover novel treatments for glioma-related diseases. ${ }^{17}$ It has been demonstrated that miR-503 is involved in angiogenesis during hypoxia, diabetes mellitusinduced impairment, endometriosis, and other diseases. ${ }^{18-20}$ Vascular endothelial growth factor (VEGF) has been found as a major contributor to angiogenesis, which induced proliferation and migration of plated endothelial cells, eventually forming tube structures. ${ }^{21}$ It was found here that VEGFA expression was downregulated in glioma cells with miR-503 overexpression (Figure 1A-C). The previous study proved that miR-503 acted as a tumor suppressor to participate in the growth and invasion of glioma cells via regulating L1CAM. ${ }^{9}$ However, the possible molecular regulation mechanism of miR-503 in the angiogenesis of glioma cells still has not been reported. In this study, the angiogenesis regulated by miR-503 in glioma cells was analyzed. Moreover, the capacity of cell proliferation, migration and angiogenesis were enhanced when miR-503 inhibited, and attenuated when miR-503 overexpression (Figure 1D-F). 
A

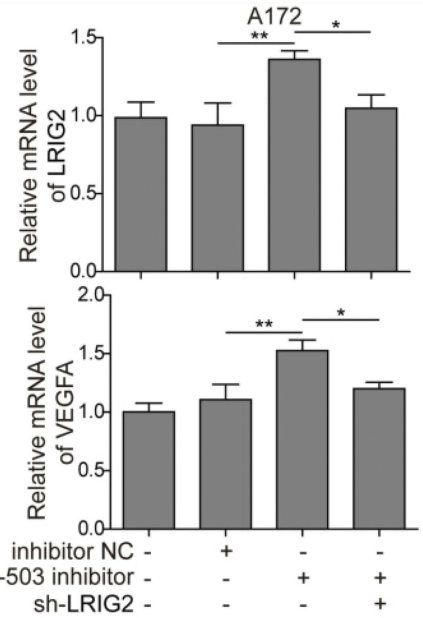

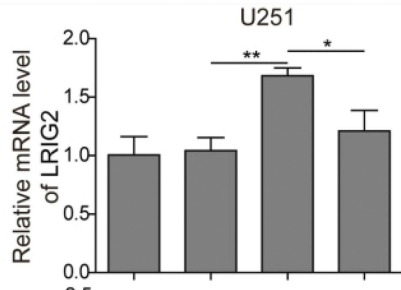

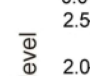

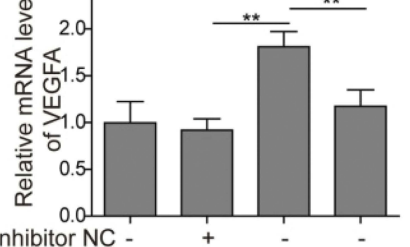

inhibitor NC miR-503 inhibitor -

sh-LRIG2 -

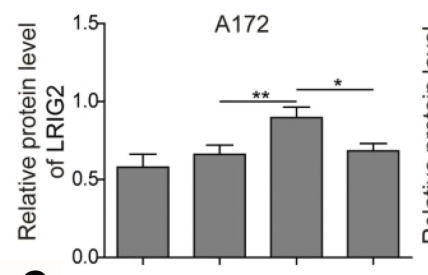

C

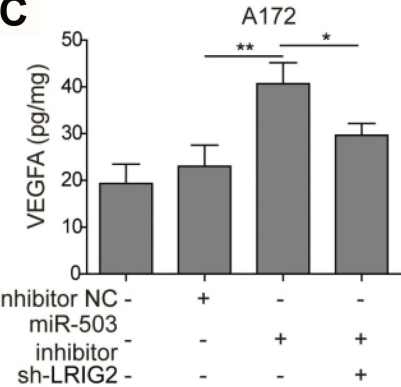

E $^{\text {Sh-LRIG2- }}$

E
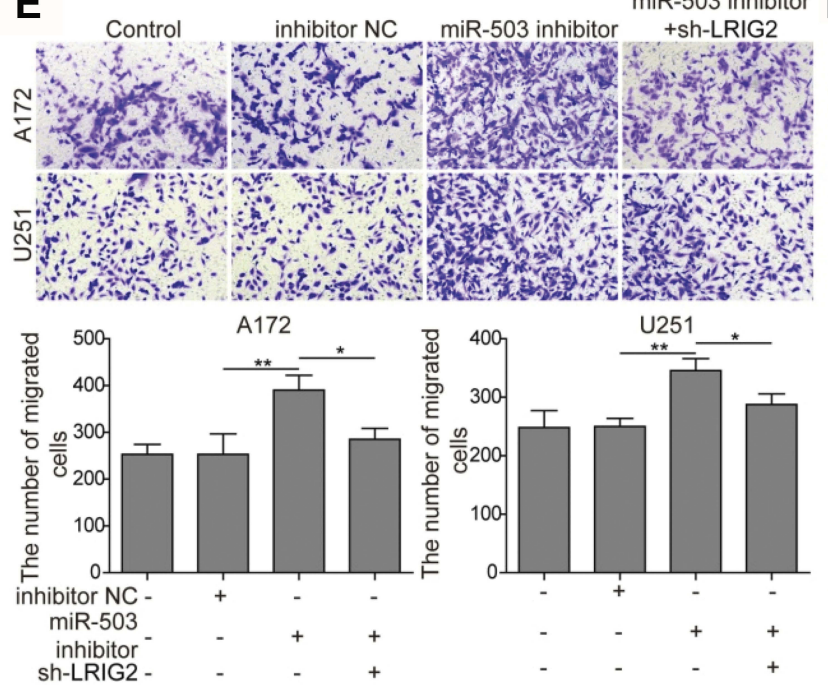

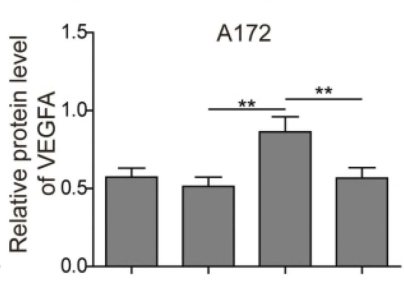

U251

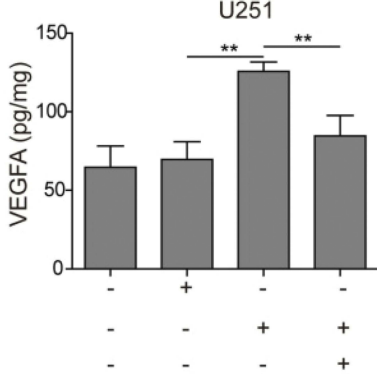

B

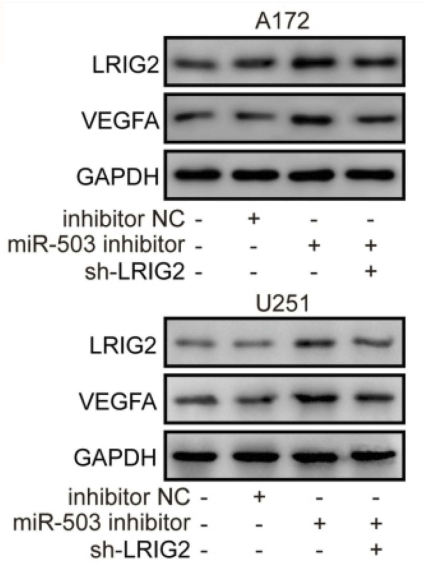

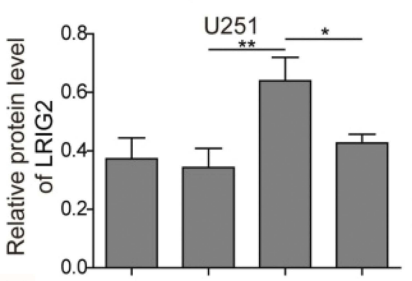

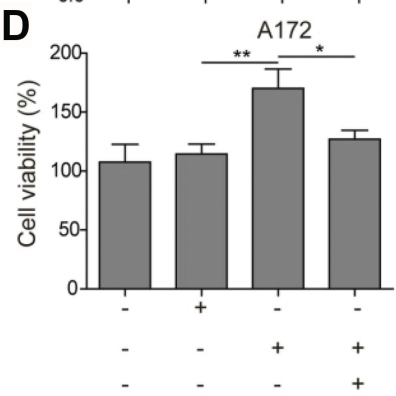

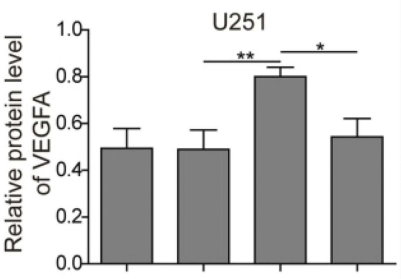

U251

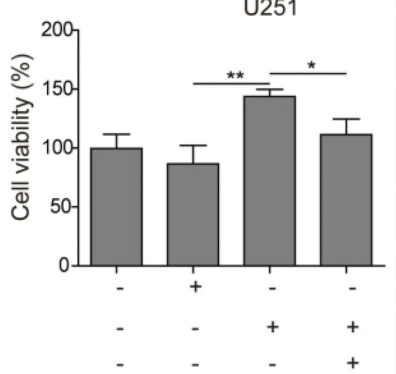

miR-503 inhibitor

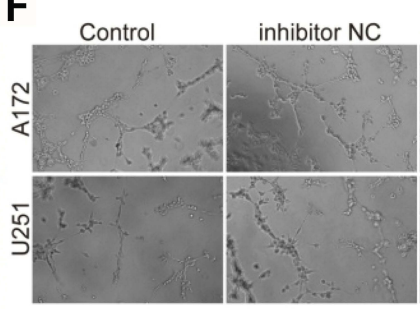

miR-503 inhibitor +sh-LRIG2
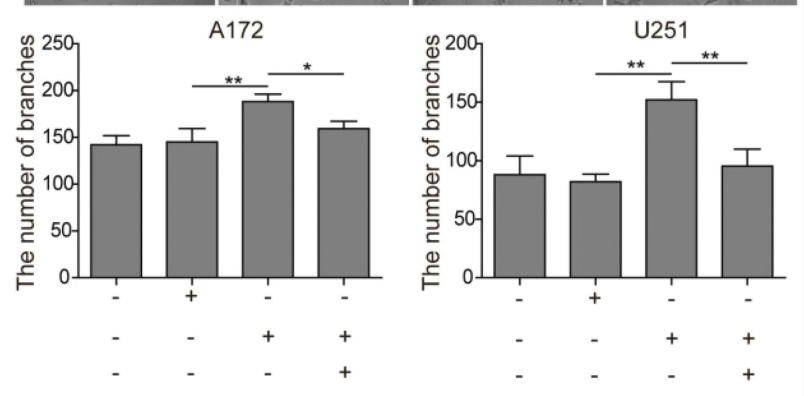

Figure 3 The effects of LRIG2 knockdown on miR-503 inhibition induced angiogenesis promotion. (A and B) The expression levels of LRIG2 and VEGFA mRNA and protein were measured by qRT-PCR and Western blot assay. (C) LRIG2 knockdown inhibits the enhanced concentration of VEGFA detected by ELISA assay. (D) The HCMEC/D3 cell proliferation ability was measured by the MTT assay in cells with miR-503 inhibitor transfection and miR-503 inhibitor+sh-LRIG2 co-transfection. (E) HCMEC/D3 migration capacity induced by coculture with glioma cells was detected by transwell assay using cocultured system. (F) Angiogenesis ability of HCMEC/D3 cells with miR-503 inhibitor transfection and miR-503 inhibitor + sh-LRIG2 co-transfection was measured. The experiment was repeated for three times. P value was determined by ANOVA following Tukey's post hoc test. Data are the mean $\pm \mathrm{SD}$ for three independent experiments. $* \mathrm{P}<0.05$ and $* * \mathrm{P}<0.01$. 

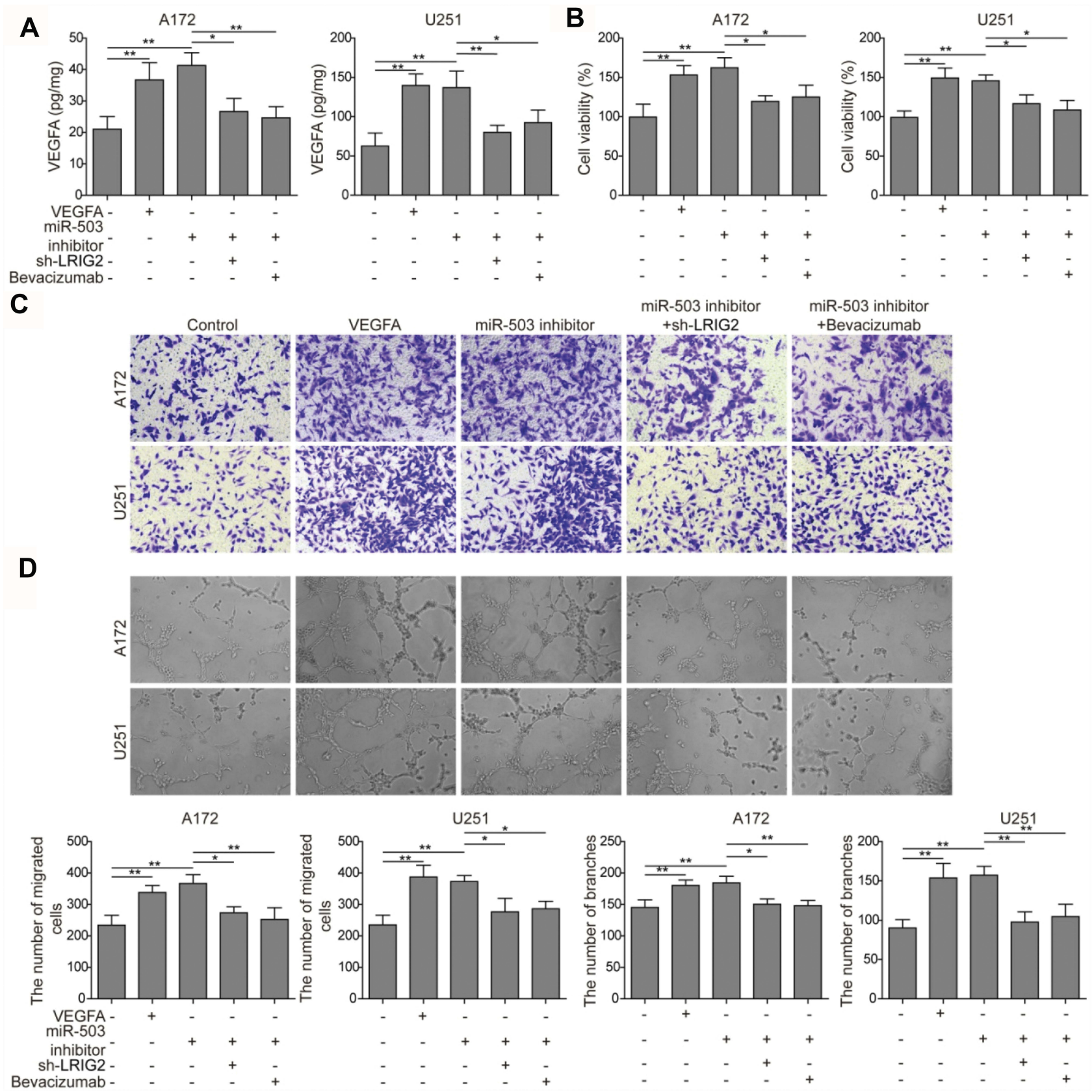

Figure 4 miR-503 regulates tumor angiogenesis via LRIG2 regulated VEGFA expression. (A) VEGFA accumulation was detected by ELISA assay in the presence of recombinant VEGFA protein, miR-503 inhibitor, sh-LRIG2, and bevacizumab. (B) MTT method was performed to determine HCMEC/D3 cell proliferation. (C and D) The cell migration and angiogenesis of HCMEC/D3 cells with miR-503 inhibitor transfection, LRIG2 knockout and bevacizumab addition were detected by transwell assay and tube formation assay, respectively. The experiment was repeated three times. Data are the mean \pm SD for three independent experiments. $P$ value was determined by ANOVA following Tukey's post hoc test. $* \mathrm{P}<0.05$ and $* * \mathrm{P}<0.01$.

These results provided an investigation suggesting that miR503 suppressed VEGFA expression during angiogenesis.

LRIG2 promotes platelet-derived growth factor (PDGF)induced glioma and involved in the regulation of angiogenesis in glioma via the EGFR/VEGFA signaling pathway. ${ }^{6,22,23}$ In this work, it was identified that miR-503 directly bound to LRIG2 by using Dual-Luciferase reporter assay (Figure 2A and B). Besides, the expression of LRIG2 was upregulated in cells with miR-503 inhibition and reduced in cells with miR-503 mimics transfection (Figure 2C and D). These data establish LRIG2 as a direct downstream target of miR-503 in glioma cells. A previous study has demonstrated that VEGFA binds to VEGFR; stimulates cell proliferation, cell migration, and tubelike structure formation of vascular endothelial cells; and ultimately promotes tumor angiogenesis. ${ }^{24}$ Meanwhile, anti-VEGF/VEGFR therapy has 
been found to markedly prolong patients' survival with nonsmall-cell lung cancer and breast cancer. ${ }^{25,26}$ Therefore, the inhibition of EGFR/VEGF-A, which acts as an essential target in tumor angiogenesis, is considered to be an effective treatment for glioma. Here, we found that in HCMEC/D3 cells with miR-503 inhibition, the cell proliferation, migration, and angiogenesis ability were enhanced. Whereas co-transfection of sh-LRIG2 and miR-503 inhibitor inhibited the effects (Figure 3A-F). It is suggested that miR-503 in human glioma might be related to tumor angiogenesis by regulating LRIG2 expression.

It has been demonstrated that miR-503 can directly target 3'-UTRs of VEGFA and regulates its mRNA and protein expression. ${ }^{11}$ Evidence has presented that miR-503 inhibits tumor growth and angiogenesis in human umbilical vein endothelial cells by downregulating FGF2 and VEGFA expression. ${ }^{13,24,27}$ A previous study has reported that miR503 regulates the release of VEGF in COPD. ${ }^{28}$ Our results confirmed that miR-503 inhibitor promoted glioma proliferation, migration, angiogenesis, and VEGFA expression; however, bevacizumab, as a VEGF inhibitor, could significantly reverse the above changes induced by miR-503 inhibitor, indicating that miR-503 regulates glioma proliferation, migration, and angiogenesis through VEGFA (Figure 4A-D). In addition, a series of molecular and genetic analyses have indicated that LRIG2 plays an important role in glioma through ERGF/VEGFA pathway, and the expression of LRIG2 is related to angiogenesis. ${ }^{6,29}$ Downregulation of LRIG2 inhibits glioma cell growth by decreasing EGFR phosphorylation and VEGFA expression. ${ }^{6}$ Our results showed that knockdown of LRIG2 could attenuate the effects of miR503 inhibitor on glioma cell function and VEGFA expression, suggesting that knockdown of miR-503 could promote glioma proliferation, migration, and angiogenesis via targeting LRIG2 to increase VEGFA expression (Figure 4A-D). These findings imply that miR-503 not only inhibits glioma proliferation, migration, and angiogenesis by directly regulating VEGFA but also repressed the above glioma function by targeting LRIG2 to regulate VEGFA expression. LRIG2 may be a new marker of glioma angiogenesis, targeting LRIG2 is expected to provide a new therapeutic strategy for the future treatment of glioma.

In conclusion, our studies suggest that by regulating VEGFA expression, miR-503 provides a mechanistic explanation as to how the regulation of cell proliferation, migration, and angiogenesis of glioma cells is achieved. This is partially accomplished by the involvement of miR-503 for LRIG2 inhibition, while LRIG2 inhibition decreased the expression of VEGFA, which in turn mediates the angiogenesis of glioma cells. Taken together, these results demonstrate that the miR-503-modulated signaling pathway is responsible for the decreased expression of VEGFA, leading to the antiangiogenesis of glioma cells, providing new insights into the signaling cascades that modulate glioma cell angiogenesis.

\section{Abbreviations}

VEGFA, vascular endothelial growth factor A; EGFR, epidermal growth factor receptor; HCMEC/D3, immortalized human cerebral microvascular endothelial cell line D3; TCM, tumor-conditioned medium; LRIG2, leucinerich repeats and immunoglobulin-like domains 2 .

\section{Disclosure}

The authors report no conflicts of interest in this work.

\section{References}

1. Zhang D, Liu Z, Zheng N, et al. MiR-30b-5p modulates glioma cell proliferation by direct targeting MTDH. Saudi J Biol Sci. 2018. S1319562X18300500. doi:10.1016/j.sjbs.2018.02.015

2. Jia P, Cai H, Liu X, et al. Long non-coding RNA H19 regulates glioma angiogenesis and the biological behavior of glioma-associated endothelial cells by inhibiting microRNA-29a. Cancer Lett. 2016. S0304383516304736. doi:10.1016/j.canlet.2016.08.009

3. Zhihua C, Zhenshengnan L, Ke M, et al. Long non-coding RNA XIST promotes glioma tumorigenicity and angiogenesis by acting as a molecular sponge of miR-429. J Cancer. 2017;8(19):4106-4116. doi:10.7150/jca.21024

4. Holmlund C, Nilsson J, Guo D, et al. Characterization and tissue-specific expression of human LRIG2. Gene (Amsterdam). 2004;332:0-43. doi:10.1016/j.gene.2004.02.002

5. Wang B, Han L, Chen R, et al. Down-regulation of LRIG2 expression by RNA interference inhibits glioblastoma cell (GL15) growth, causes cell cycle redistribution, increases cell apoptosis and enhances cell adhesion and invasion in vitro. Cancer Biol Ther. 2009;8 (11):1018-1023. doi:10.4161/cbt.8.11.8375

6. Yang H-K, Chen H, Mao F, et al. Downregulation of LRIG2 expression inhibits angiogenesis of glioma via EGFR/VEGF-A pathway. Oncol Lett. 2017;14(4):4021. doi:10.3892/ol.2017.6671

7. Wang Y, Lee CGL. MicroRNA and cancer-focus on apoptosis. J Cell Mol Med. 2009;13(1):12. doi:10.1111/j.1582-4934.2008.00510.x

8. Shea A, Harish V, Afzal Z, et al. MicroRNAs in glioblastoma multiforme pathogenesis and therapeutics. Cancer Med. 2016;5 (8):1917-1946.

9. Liu H, Song Z, Liao D, et al. miR-503 inhibits cell proliferation and invasion in glioma by targeting L1CAM. Int J Clin Exp Med. 2015;8 (10): 18441 .

10. Li B, Liu L, Li X, et al. miR-503 suppresses metastasis of hepatocellular carcinoma cell by targeting PRMT1. Biochem Biophys Res Commun. 2015;464(4):S0006291X15302266. doi:10.1016/j.bbrc.20 15.06.169

11. Zhou B, Ma R, Si W, et al. MicroRNA-503 targets FGF2 and VEGFA and inhibits tumor angiogenesis and growth. Cancer Lett. 2013;333 (2):159-169. doi:10.1016/j.canlet.2013.01.028

12. Carmeliet P, Jain RK. Molecular mechanisms and clinical applications of angiogenesis. Nature. 2011;473(7347):298-307. doi:10.1038/nature10144 
13. Cross MJ. FGF and VEGF function in angiogenesis: signalling pathways, biological responses and therapeutic inhibition. Trends Pharmacol Sci. 2001;22(4):201-207. doi:10.1016/S0165-6147(00)01676-X

14. Zhang Y, Guo X, Xiong L, et al. MicroRNA-101 suppresses SOX9-dependent tumorigenicity and promotes favorable prognosis of human hepatocellular carcinoma. Febs Lett. 2012;586 (24):4362-4370. doi:10.1016/j.febslet.2012.10.053

15. Cucullo L, Couraud PO, Weksler B, et al. Immortalized human brain endothelial cells and flow-based vascular modeling: a marriage of convenience for rational neurovascular studies. J Cereb Blood Flow Metab. 2007;28(2):312-328. doi:10.1038/sj.jcbfm.9600525

16. Hatherell K, Couraud P-O, Romero IA, et al. Development of a three-dimensional, all-human in vitro model of the blood-brain barrier using mono-, co-, and tricultivation transwell models. J Neuro Methods. 2011;199:223-229. doi:10.1016/j.jneumeth.2011.05.012

17. Yu D, Han G, Liu H, Gao L, Verma V. Treatment of adult brainstem glioma with combined antiangiogenic therapy: a case report and literature review. Onco Targets Ther. 2018;12:1333-1339.

18. Wen Y, Chen R, Zhu C, et al. MiR-503 suppresses hypoxia-induced proliferation, migration and angiogenesis of endothelial progenitor cells by targeting Apelin. Peptides. 2018;105. doi:10.1016/j.peptides.2018.05.008

19. Caporali A, Meloni M, Vollenkle C, et al. Deregulation of microRNA-503 contributes to diabetes mellitus-induced impairment of endothelial function and reparative angiogenesis after limb ischemia. Circulation. 2011;123(3):282-291. doi:10.1161/CIRCULATIONAHA.110.952325

20. Hirakawa T, Nasu K, Abe W, et al. miR-503, a microRNA epigenetically repressed in endometriosis, induces apoptosis and cell-cycle arrest and inhibits cell proliferation, angiogenesis, and contractility of human ovarian endometriotic stromal cells. Hum Reprod. 2016;31 (11):2587-2597. dew217v1
21. Prior BM. What makes vessels grow with exercise training? $J$ Appl Physiol. 2004;97(3):1119-1128. doi:10.1152/japplphysiol.00035.2004

22. Holmlund C, Haapasalo H, Yi W, et al. Cytoplasmic LRIG2 expression is associated with poor oligodendroglioma patient survival. Neuropathology. 2009;29(3):242-247. doi:10.1111/neu.2009.29.issue-3

23. Holmlund C, Karlsson T, Borota OC, et al. Abstract 1133: LRIG2 promotes PDGF induced experimental glioma. Cancer Res. 2011;71 (8 Supplement): 1133 .

24. Larsen AK, Ouaret D, Ouadrani KE, et al. Targeting EGFR and VEGF(R) pathway cross-talk in tumor survival and angiogenesis. Pharmacol Ther. 2011;131(1):80-90. doi:10.1016/j.pharmthera.2011.03.012

25. Jiang T, Qiao M, Zhou F, et al. Effect of combined therapy inhibiting EGFR and VEGFR pathways in non-small-cell lung cancer on progression-free and overall survival. Clin Lung Cancer. 2017;18 (4):421.e3-431.e3. S1525730416303886.

26. Yu X, Li W, Deng Q, et al. Neoalbaconol inhibits angiogenesis and tumor growth by suppressing EGFR-mediated VEGF production. Mol Carcinog. 2017;56(5):1414-1426. doi:10.1002/mc.v56.5

27. Przybylski M. A review of the current research on the role of bFGF and VEGF in angiogenesis. J Wound Care. 2009;18(12):516-519. doi:10.12968/jowc.2009.18.12.45609

28. Ikari J, Nelson AJ, Obaid J, et al. Reduced microRNA-503 expression augments lung fibroblast VEGF production in chronic obstructive pulmonary disease . PLoS One. 2017;12(9):e0184039. doi:10. 1371/journal.pone.0184039

29. Martens T. Inhibition of glioblastoma growth in a highly invasive nude mouse model. Clin Cancer Res. 2008;14(17):5447-5458. doi:1 0.1158/1078-0432.CCR-08-0147

\section{Publish your work in this journal}

Cancer Management and Research is an international, peer-reviewed open access journal focusing on cancer research and the optimal use of preventative and integrated treatment interventions to achieve improved outcomes, enhanced survival and quality of life for the cancer patient.
The manuscript management system is completely online and includes a very quick and fair peer-review system, which is all easy to use. Visit http://www.dovepress.com/testimonials.php to read real quotes from published authors. 